\title{
Comparing Two Proximal Measures of Unrecognized Clinical Deterioration in Children
}

\begin{abstract}
Tina Sosa, MD*, Sarah Ferris, BA¹, Carol Frese, RN², Deborah Hacker, RN², Maya Dewan, MD, MPH ${ }^{3,4,5}$, Patrick W Brady, MD, MSc ${ }^{1,5,6}$
'Division of Hospital Medicine, Cincinnati Children's Hospital Medical Center, Cincinnati, Ohio; ${ }^{2}$ Department of Patient Services, Cincinnati Children's Hospital Medical Center, Cincinnati, Ohio; ${ }^{3}$ Division of Critical Care Medicine, Cincinnati Children's Hospital Medical Center, Cincinnati, Ohio; ${ }^{4}$ Division of Biomedical Informatics, Cincinnati Children's Hospital Medical Center, Cincinnati, Ohio; ${ }^{5}$ Department of Pediatrics, University

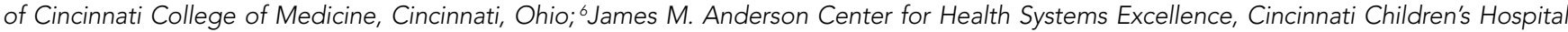
Medical Center, Cincinnati, Ohio.
\end{abstract}

Critical deterioration events (CDEs) and emergency transfers (ETs) are two proximal measures to cardiopulmonary arrest, and both aim to evaluate how systems recognize and respond to clinical deterioration in children. This retrospective observational study sought to (1) characterize CDEs and ETs by timing, overlap, and intervention category, and (2) evaluate the performance of the watcher identification system and the pediatric early warning score (PEWS) to identify patients who experience these events. A total of $359 \mathrm{CDEs}$ and $88 \mathrm{ETs}$ occurred during the study period. Respiratory events were most common and accounted for $80.5 \%$ of CDEs and $47.7 \%$ of ETs. A narrow majority of patients were identified as watchers (55.4\% of CDEs and $51.1 \%$ of ETs). In total, $85.5 \%$ of CDEs and $87.5 \%$ of ETs were identified as watchers, elevated PEWS, or both. Opportunities exist for improved escalation plans for high-risk patients to prevent the need for emergent intervention. Journal of Hospital Medicine 2020;15:673-676. (C) 2020 Society of Hospital Medicine $\bigcup$ nrecognized in-hospital clinical deterioration can lead to substantial morbidity and mortality. ${ }^{1}$ As a result, hospitals have implemented systems to identify and mitigate this form of potentially preventable harm..$^{2-4}$ Cardiopulmonary arrest rates are useful metrics to evaluate the effectiveness of systems designed to identify and respond to deteriorating adult patients. ${ }^{5}$ Pediatric arrests outside of the intensive care unit (ICU) are rare; therefore, the identification of valid and more frequent proximal measures of deterioration is critical to the assessment of current systems and to guide future improvement efforts. ${ }^{6}$

Bonafide et al developed and validated the critical deterioration event (CDE) metric, demonstrating that children who were transferred to the ICU and who received noninvasive ventilation, intubation, or vasopressor initiation within 12 hours of transfer had an over 13-fold increased risk of in-hospital mortality. ${ }^{7}$ Implementation of a rapid response system was subsequently associated with a decrease in the trajectory of CDEs. ${ }^{2}$ At Cincinnati Children's Hospital Medical Center (CCHMC), an additional proximal outcome measure was developed for unrecognized clinical deterioration: emergency transfers (ETs). ${ }^{8,9}$ An event meets criteria for an ET when the

*Corresponding Author: Tina Sosa, MD; Email: Tina.sosa@cchmc.org; Twitter: @TinaKSosa.

Published online first October 21, 2020

Received: April 24, 2020; Revised: July 31, 2020; Accepted: August 4, 2020

() 2020 Society of Hospital Medicine DOI 10.12788/jhm.3515 patient undergoes intubation, inotropic support, or three or more fluid boluses in the first hour after arrival or prior to ICU transfer. ${ }^{9}$ Recently, ETs were associated with an increased in-hospital mortality, ICU length of stay, and post-transfer hospital length of stay when compared with nonemergent transfers. ${ }^{10,11}$

While both CDEs and ETs were associated with adverse outcomes in children and may be modifiable through better rapid response systems, researchers have not previously compared the extent to which CDEs and ETs capture similar versus distinct events. Furthermore, the ability of focused situation awareness interventions to identify high-risk patients has not previously been assessed. Situation awareness is defined as the perception of elements in the environment, the comprehension of their meaning, and the projection of their status in the near future. ${ }^{12}$ Clinically, improved situation awareness can lead to earlier recognition of deterioration and a reduction in failure to rescue events. ${ }^{9}$ The objectives of this study were to (1) describe CDEs and ETs and assess for similarities, differences, and trends, and (2) evaluate the utility of situation awareness interventions to detect patients who experience these events.

\section{METHODS}

\section{Setting and Inclusion Criteria}

We conducted a retrospective cross-sectional study at CCHMC, a free-standing tertiary care children's hospital. We included all patients cared for outside of the ICU during their hospitalization from January 2016 to July 2018. Transfer to the ICU included the pediatric and the cardiac ICUs. 
TABLE. Categorization of Proximal Deterioration Metrics and Identification by Situation Awareness Interventions

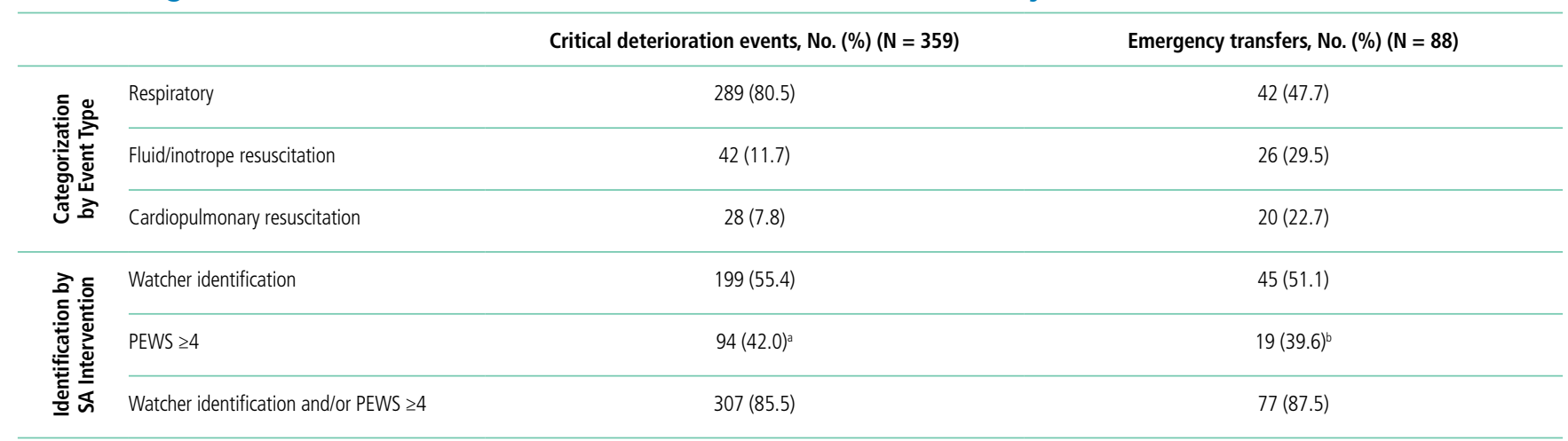

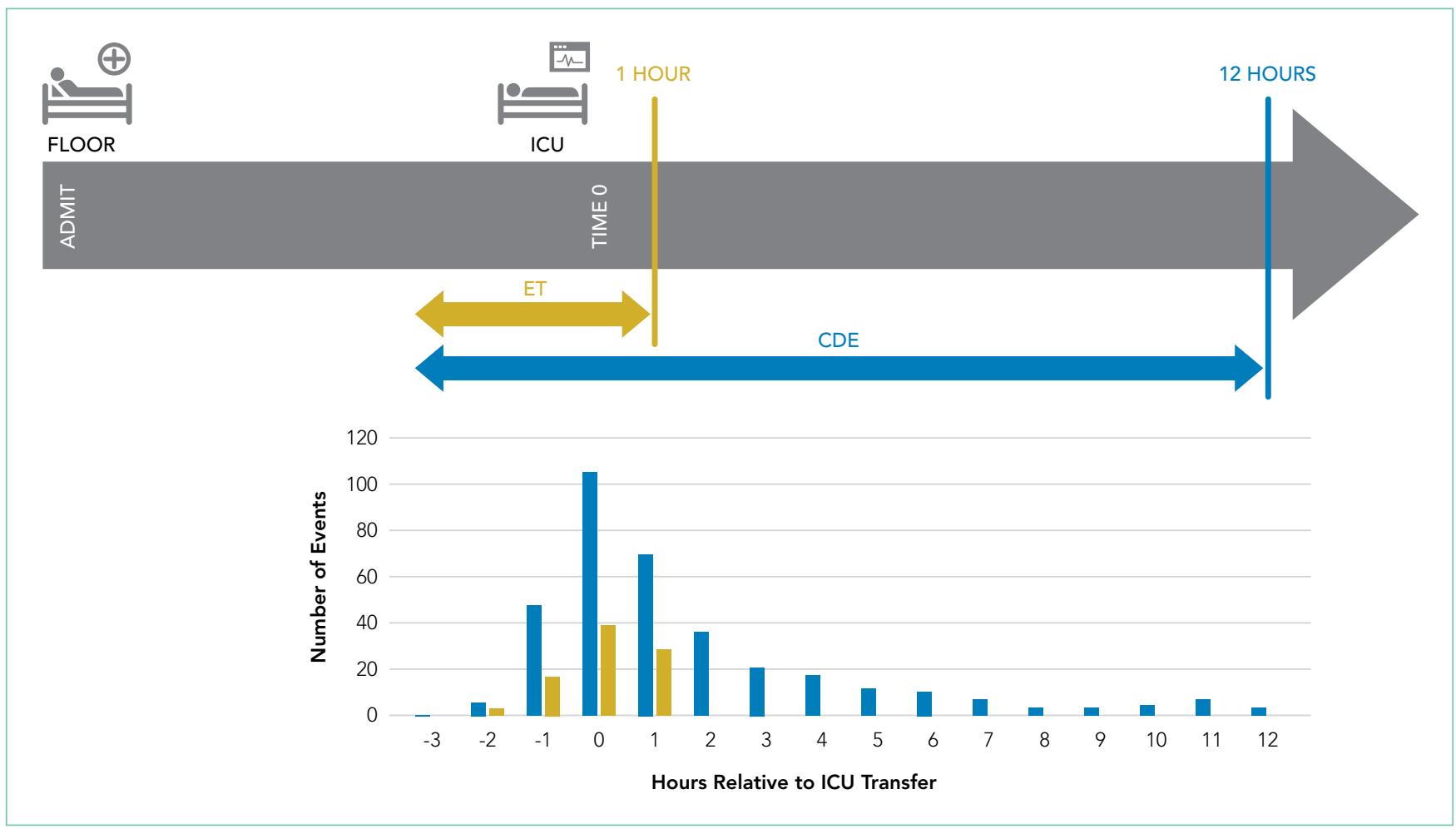

FIG. Visual Representation and Timing of Proximal Measures of Clinical Deterioration in Children. All events that occurred prior to ICU transfer (time 0) were (a) respiratory events in which the patient required positive pressure ventilation prior to arrival to the ICU or (b) cardiopulmonary arrest events in which resuscitation was initiated on the floor.

Abbreviations: $\mathrm{CDE}$, critical deterioration event; $\mathrm{ET}$, emergency transfer; ICU, intensive care unit.

\section{Study Definitions}

CDEs were events in which a patient received noninvasive ventilation, intubation, or vasopressor initiation within 12 hours of ICU transfer (Figure). ${ }^{7}$ ETs were events in which a patient underwent intubation, inotropes, or three or more fluid boluses in the first hour after arrival or before transfer (Figure). ${ }^{9}$ We examined two distinct situation awareness interventions: watcher identification and the pediatric early warning score (PEWS). A watcher is a situation awareness concern based on clinician perception, or "gut feeling, " that the patient is at high risk for deterioration. ${ }^{9,13}$ When clinicians designate a patient as a watcher in the electronic medical record, they establish an action plan, reassessment timeline, and objective criteria for activation of the rapid response team to assess the patient. Watcher patients are discussed at institution-wide safety huddles three times daily. The PEWS is a reproducible assessment of the patient's status based on physiologic parameters, including behavior, cardiovascular, and respiratory assessments. ${ }^{3,4}$ At CCHMC, a Monaghan PEWS score is calcu- 
lated with each assessment of vital signs. ${ }^{14}$ The bedside nurse calls the physician or advanced practice provider to assess the patient for a score of 4 or greater.

\section{Event Identification and Classification}

Two trained research nurses (C.F. and D.H.) manually reviewed all ICU transfers during the study period to determine if CDE criteria were met. Events meeting CDE criteria were classified as respiratory (requiring noninvasive or invasive ventilation), cardiac (requiring inotropes), or cardiopulmonary resuscitation (CPR) in which cardiac and respiratory interventions were initiated simultaneously. Additional information obtained included the time the patient met CDE criteria relative to the time of ICU transfer, watcher identification prior to the event, and the highest PEWS documented within 12 hours of the event. A physician (T.S.) performed manual chart review of each CDE as an additional validation step. ETs during the study period were obtained from an existing institutional database. ICU transfers meeting ET criteria are entered into this database in nearly real time by the inpatient nurse manager; this nurse attends all rapid response team calls and is aware of the disposition for each event. A physician (T.S.) performed manual chart review of each ET to determine event classification by intervention type, watcher identification, and the highest PEWS documented within 12 hours of the event. All CDEs and ETs were cross-referenced to determine overlap.

\section{Outcome Measures and Statistical Analysis}

The primary outcomes were CDEs and ETs, calculated as absolute counts and number of events per 10,000 non-ICU patient days. Events were classified by (1) category of intervention, (2) watcher identification prior to the event, and (3) PEWS of 4 or greater documented in the 12 hours prior to the event.

\section{RESULTS}

\section{Incidence and Overlap of CDEs and ETs}

There were 1,828 ICU transfers during the study period, of which 365 (20\%) met criteria for a CDE, ET, or both. Among events captured, 359 (98.4\%) met criteria for a CDE, occurring at a rate of 16.7 per 10,000 non-ICU patient days, and 88 (24.1\%) met criteria for an ET, occurring at a rate of 4.1 per 10,000 non-ICU patient days (Table). Of the $88 \mathrm{ETs}, 82$ also met criteria for a CDE.

\section{Timing and Categorization of CDEs and ETs}

Despite the 12-hour time horizon, most CDEs (62.1\%) met criteria within 1 hour of ICU transfer, and $79.9 \%$ met criteria within 3 hours (Figure). Respiratory events were most common for both CDEs (80.5\%) and ETs (47.7\%) (Table). Of respiratory CDEs, $67.4 \%$ required noninvasive ventilation, and $32.5 \%$ required invasive ventilation. Fluid or inotrope support were responsible for $11.7 \%$ of CDEs and nearly one-third of ETs; of note, the CDE definition does not include fluid boluses. Less than $10 \%$ of CDEs were characterized by CPR, whereas this accounted for $22.7 \%$ of ETs.

\section{Identification of Events by Situation Awareness Interventions}

The Table depicts the identification of events by watcher status and PEWS. All events were included for watcher identification, and events with a documented score in the 12 hours prior to transfer were included for PEWS. While half or less of the events were captured by watcher or PEWS separately, over $85 \%$ of events were captured by either one or both of the situation awareness interventions. The situation awareness interventions identified CDEs and ETs similarly.

\section{DISCUSSION}

This study is the first to classify and compare two proximal measures of clinical deterioration in children. Given that children with escalating respiratory symptoms are often treated successfully outside of the ICU, the findings that most events are respiratory in nature and occur within 1 hour of transfer are not unexpected. The analysis of situation awareness interventions suggests that neither watcher identification nor PEWS is independently sufficient to predict future deterioration. These findings support the necessity of both a clinician "gut feeling" and objective vital sign and physical exam findings to indicate a patient's clinical status. ${ }^{9}$ Initiatives to improve the early recognition and mitigation of patient deterioration should focus on both tools to initiate an escalation of care, and work to understand gaps in these identification systems, which currently miss approximately $15 \%$ of acutely deteriorating patients. Although most patients had watcher identification or elevated PEWS prior to the event, they still required emergent life-sustaining care, which suggests that opportunities exist to improve mitigation and escalation pathways as a critical prevention effort. ${ }^{7,10}$

It is likely that CDEs and ETs are important outcome metrics in the evaluation of pediatric escalation systems, including rapid response systems. ${ }^{15}$ ETs are less common and more specific for unrecognized deterioration, which makes them a more feasible early metric for assessment. CDEs, which are likely more sensitive, may be useful in settings in which deterioration is rare or a more common outcome enhances power to detect the effect of interventions. ${ }^{10}$

This study has limitations and lends itself to future work. While CDEs and ETs are more common than cardiopulmonary arrest, they remain relatively uncommon. This was a single-site study at a large, tertiary care, free-standing children's hospital, so generalizability to centers with different characteristics and patient populations may be limited. Future work should focus on comparing patient-level outcomes of CDEs and ETs, including length of stay and mortality. The determination of specific diagnoses and conditions associated with CDEs and ETs may inform targeted preventive improvement science interventions.

\section{CONCLUSION}

CDEs were roughly fourfold more common than ETs, with most CDEs occurring within 1 hour of ICU transfer. Most patients were identified by either watcher status or elevated PEWS, suggesting that these tools, when utilized as complementary 
situation awareness interventions, are important for identifying patients at risk for deterioration. Opportunities exist for improved escalation plans for patients identified as high-risk to prevent the need for emergent life-sustaining intervention.

Disclosures: The authors have no conflicts of interest to disclose.

Funding: Dr Brady receives career development support from Agency for Healthcare Research and Quality K08-HS023827. The project described was supported by the National Center for Advancing Translational Sciences of the National Institutes of Health, under Award Number 5UL1TR001425-04. The content is solely the responsibility of the authors and does not necessarily represent the official views of the $A H R Q$ or $\mathrm{NIH}$.

\section{References}

1. Buist M, Bernard S, Nguyen TV, Moore G, Anderson J. Association between clinically abnormal observations and subsequent in-hospital mortality: a prospective study. Resuscitation. 2004;62(2):137-141. https://doi.org/10.1016/j. resuscitation.2004.03.005

2. Bonafide $C P$, Localio $A R$, Roberts $K E$, Nadkarni VM, Weirich $C M$, Keren R. Impact of rapid response system implementation on critical deterioration events in children. JAMA Pediatr. 2014;168(1):25-33. https://doi.org/10.1001/ jamapediatrics.2013.3266

3. Duncan H, Hutchison J, Parshuram CS. The Pediatric Early Warning System score: a severity of illness score to predict urgent medical need in hospitalized children. J Crit Care. 2006;21(3):271-278. https://doi.org/10.1016/j. jcrc.2006.06.007

4. Sefton G, McGrath C, Tume L, Lane S, Lisboa PJ, Carrol ED. What impact did a Paediatric Early Warning system have on emergency admissions to the paediatric intensive care unit? an observational cohort study. Intensive Crit Care Nurs. 2015;31(2):91-99. https://doi.org/10.1016/j.iccn.2014.01.001
5. Schein RM, Hazday N, Pena M, Ruben BH, Sprung CL. Clinical antecedents to in-hospital cardiopulmonary arrest. Chest. 1990;98(6):1388-1392. https:// doi.org/10.1378/chest.98.6.1388

6. Feudtner C, Berry JG, Parry G, et al. Statistical uncertainty of mortality rates and rankings for children's hospitals. Pediatrics. 2011;128(4):e966-e972. https://doi.org/10.1542/peds.2010-3074

7. Bonafide $\mathrm{CP}$, Roberts $\mathrm{KE}$, Priestley MA, et al. Development of a pragmatic measure for evaluating and optimizing rapid response systems. Pediatrics. 2012;129(4):e874-e881. https://doi.org/10.1542/peds.2011-2784

8. Brady PW, Goldenhar LM. A qualitative study examining the influences on situation awareness and the identification, mitigation and escalation of recognised patient risk. BMJ Qual Saf. 2014;23(2):153-161. https://doi. org/10.1136/bmjqs-2012-001747

9. Brady PW, Muething S, Kotagal U, et al. Improving situation awareness to reduce unrecognized clinical deterioration and serious safety events. Pediatrics. 2013;131(1):e298-e308. https://doi.org/10.1542/peds.2012-1364

10. Hussain FS, Sosa T, Ambroggio L, Gallagher R, Brady PW. Emergency transfers: an important predictor of adverse outcomes in hospitalized children. J Hosp Med. 2019;14(8):482-485. https://doi.org/10.12788/jhm.3219

11. Aoki Y, Inata Y, Hatachi T, Shimizu Y, Takeuchi M. Outcomes of 'unrecognised situation awareness failures events' in intensive care unit transfer of children in a Japanese children's hospital. J Paediatr Child Health. 2019;55(2):213-215. https://doi.org/10.1111/jpc.14185

12. Endsley MR. Toward a theory of situation awareness in dynamic systems. Human Factors. 1995;37(1):32-64. https://doi.org/10.1518/001872095779049543

13. McClain Smith M, Chumpia M, Wargo L, Nicol J, Bugnitz M. Watcher initiative associated with decrease in failure to rescue events in pediatric population. Hosp Pediatr. 2017;7(12):710-715. https://doi.org/10.1542/hpeds.2017-0042

14. Monaghan A. Detecting and managing deterioration in children. Paediatr Nurs. 2005;17(1):32-35. https://doi.org/10.7748/paed2005.02.17.1.32.c964

15. Subbe CP, Bannard-Smith J, Bunch J, et al. Quality metrics for the evaluation of Rapid Response Systems: proceedings from the third international consensus conference on Rapid Response Systems. Resuscitation. 2019;141:112. https://doi.org/10.1016/j.resuscitation.2019.05.012 